\title{
Effect of Wall Variable Thickness on Patient Specific Finite Element Abdominal Aortic Aneurysm Models
}

\author{
Omar Altwijri \\ Biomedical Technology Department, College of Applied Medical Sciences, King Saud University, Riyadh, \\ KSA \\ oaltwijri@ksu.edu.sa
}

\begin{abstract}
The aim of this study is to analyze the possibilities to measure the wall stress on the thinnest and thickest side of the AAA by using patient's Specific Finite Element (SFE) models, in order for understanding the rupture of AAA in a better approach. Patient specific model with different in position, Finite Ele-ment Analysis (FEA) models that were studied earlier by Di Martino et al. (1998) [1], was adopted. The AAA was modelled as a homogenous, isotropic, incompressible, linear elastic material with Young's modulus (E) and Poisson's ratio $(\mu)$ having value of $0.11 \mathrm{MPa}$ and $\mu=0.45$, respectively. The values of $E$ and $\mu$ was determined from uniaxial loading tests performed on specimens of AAA. In order to cast peak systolic blood pressure of a healthy patient, pressure of $145 \mathrm{mmHg}$ was directed to the internal surface of the models. FEA models of AAA were analysed using ANSYS Finite Element Package 15.0 version. Values of stress was observed on the scale of von-Mises, this method helps to locate area with highest stress i.e. to calculate failure criteria by combining the stress in threedimensions. In order to determine the collapse of AAA wall von-Mises stress is commonly use for the assessment of the AAA stress. Our findings and results strengthens the earlier studies performed by Polzer et al. (2010) [2] and Altuwaijri (2015) [3], where AAA wall stress was reduced by formation of thrombus. Thus reducing the chance of AAA rupture and supporting the wall strength. Our findings suggest that thrombus clearly works as a shield to guard the AAA wall from rupture. However, more research and further studies are required that corroborate the relation between the biological and mechanical factors to understand the role of the thrombus for AAA rupture.
\end{abstract}

Keywords: Abdominal aortic aneurysm, Wall thickness.

\section{Introduction}

Pathological dilatations of aneurysm are irreversible and enduring, that can occur in any blood vessel [4]. Aneurysm dilatations are of serious concern, when occur at the infrarenal section of the abdominal aorta. Abdominal Aorta Aneurysm (AAA) [4] , can eventually rupture if left untreated, with a mortality rate of $90 \%$ among ruptured patients [5]. Claiming around 15,000 lives annually in America [6]. AAA's are considered to be proactive when the abdominal aorta reaches upto $3 \mathrm{~cm}$ in diameter [ $\underline{6}, \underline{7}]$. Commonly, ultrasound imaging or computed tomography scans are utilize for monitoring the growth 
Omar Altwijri; Effect of Wall Variable Thickness on Patient Specific Finite Element Abdominal Aortic Aneurysm Models. Journal of Biomedical Engineering and Medical Imaging, Volume 5, No 5 October (2018), pp 6-12

rate of the diameter of abdominal aorta, in case the growth rate observed $1 \mathrm{~cm}$ per year or greater, when a AAA reaches $5.5 \mathrm{~cm}$, a reparative surgery is performed [?]. It has been noted that smaller AAAs with $5.5 \mathrm{~cm}$ can also get rupture, in contrast to larger AAAs are also found to be steady and may get pointless surgery $[\underline{5}, \underline{8-11}]$. Thus raising a concern to perform surgery on AAAs with $5.5 \mathrm{~cm}$ in diameter and a growth rate of $1 \mathrm{~cm}$ in diameter. Therefore, further studies are required to analyse the risks of expansion and rupture for AAAs [12].

Various studies have been performed noninvasively [13] and invasively [14-16] to understand the wall strength of the AAA. These experimental studies carried out to numerically comprehend the procedure for rupture of AAA [4]. As till date limited studies executed for establishing the rupture potential. As the stress of blood pressure rises on the walls of AAA, rupture caused due to weaken in strength of the wall [17]. Truijers et al. (2007) [18] and Fillinger et al. (2002) [19], studies illustrates wall stress analyses AAA from patient-specific size on a computer-based method, using Finite element method (FEM). The geometrical dimension of the AAA is ascertain from Computed Topographic Angiography [17]. Significant difference been observed in material parameters among healthy abdominal aortas and AAA wall. According to Vorp (2007) [] ], a maximum error of less than 5\% can occur if standard values for these parameters are used. With the use Ultrasound technology, measurements can be measured for superficial arteries, by using a high frequency ultrasound waves. A study performed by Haller et al. (2007) [20], for analysing carotid artery by using high frequency results in high resolution images gives exceptional possibilities.

Studies on the wall stress of AAA models by using patient SFE models are complex and scarce subject. The aim of this study is to analyze the possibilities to measure the wall stress on the thinnest and thickest side of the AAA by using patient's SFE models, in order for understanding the rupture of AAA in a better approach. To the knowledge of the author, this is the very first study to analyze wall stress at the thinnest and thickest sides of the AAA.

\section{Methodology}

To simplify the AAA and model it on a CAD software. Patient specific model with different in position (Case 1, Case 2, Case 3 and Case 4) FEA models that studied earlier by Di Martino et al. (1998) [1] , was adopted. A precise design and approach of AAA was used to model the CAD with the help of FEA for this study. Di Martino et al. (1998) [1] , obtained the specimen's dimension and size during AAA surgery. The AAA was modelled as a homogenous, isotropic, incompressible, linear elastic material with Young's modulus (E) and Poisson's ratio $(\mu)$ having value of $0.11 \mathrm{MPa}$ and $\mu=0.45$, respectively. The values of $E$ and $\mu$ was determined from uniaxial loading tests performed on specimens of AAA. In order to cast peak systolic blood pressure of a healthy patient, pressure of $145 \mathrm{mmHg}$ was directed to the internal surface of the models.

The four patient specific FEA models were sketched, extruded and analyzed using the ANSYS Finite Element Package 15.0 version (ANSYS Inc., Canonsburg, Pennsylvania, United States, and License Server 1055@PC13) at the department of Biomedical Technology, College of Applied Medical Sciences, King Saud University. The four different models are seen in Figure 1, only the thrombus thickness from inside the aneurysm varied in each model in contrast to the dimension of the models. 
Case 1 AAA thickness of the lumen of blood is at anterior position as seen in Figure 1a. In figure $1 \mathrm{~b}$, Case 2 AAA thickness of the lumen of blood is at posterior position. Case 3 AAA thickness of the lumen of blood is at right position see figure 1c. Figure $1 \mathrm{~d}$, Case 4 thickness of the lumen of the AAA can be seen at the left.

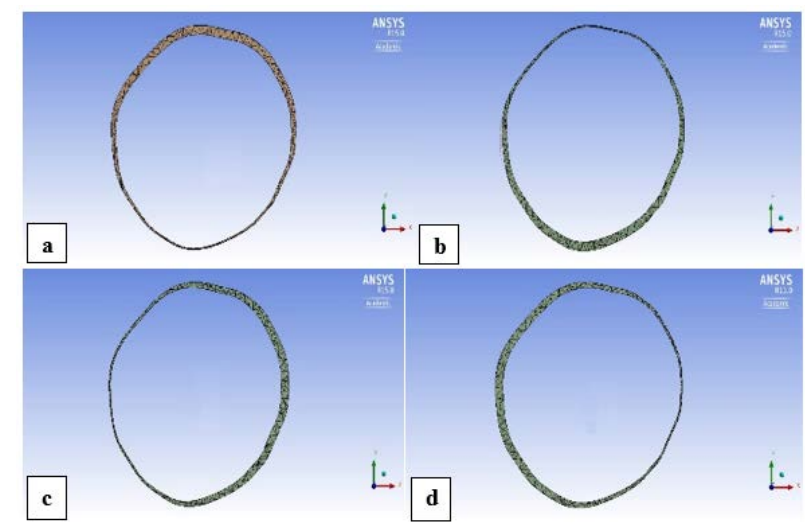

Figure 1 Models of AAA with same thickness and thinness at different positions.

Four FEA models of AAA were analysed using ANSYS Finite Element Package 15.0 version. Values of stress was observed on the scale of von-Mises, this method helps to locate area with highest stress i.e. to calculate failure criteria by combining the stress in three-dimensions. In order to determine the collapse of AAA wall von-Mises stress is commonly use for the assessment of the AAA stress.

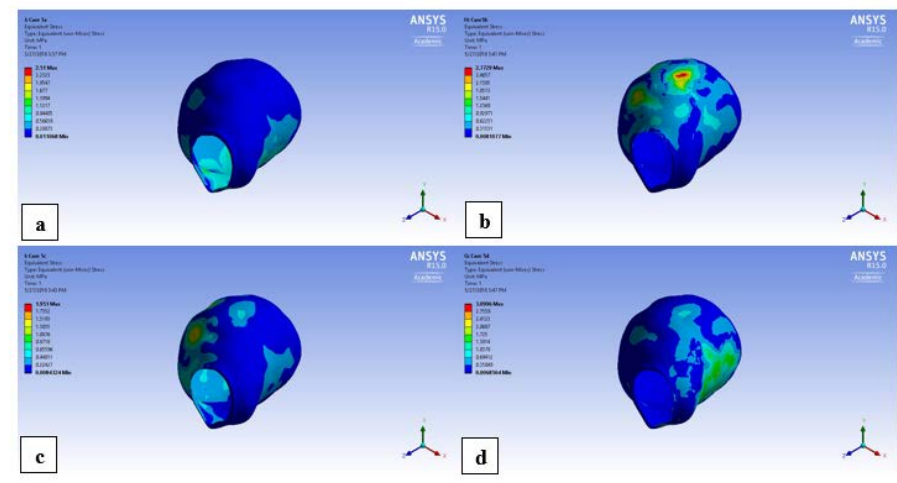

Figure 2 Effective wall stress distribution of four models at $Z$ and $X$ planes

Figure 2, displays us the four different models of AAA where von-Mises stress distribution across the walls are viewed across $Z$ and $X$ planes, over peak systolic pressure at static simulation. The maximum stress location is clearly visible in second model in figure $2 \mathrm{~b}$, with maximum stress value of $2.7729 \mathrm{MPa}$ equivalent to (von-Mises) stress, as AAA rupture is obvious to happen at the thinnest side of the wall. 


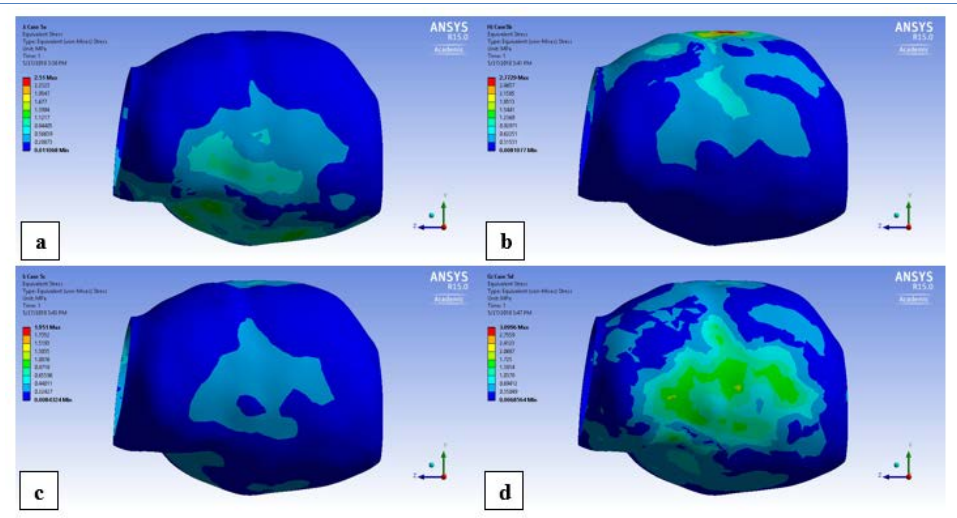

Figure 3 Effective wall stress distribution of four models at $X$ plane

In figure 3 we can see four different models of AAA where von-Mises stress distribution across the walls are viewed across $X$ plane, over peak systolic pressure at static simulation. The maximum stress location is again visible in second model (figure $3 \mathrm{~b}$ ) with maximum stress value of $2.7729 \mathrm{MPa}$ equiva-lent to (von-Mises) stress, as AAA rupture is obvious to happen at the thinnest side of the wall.

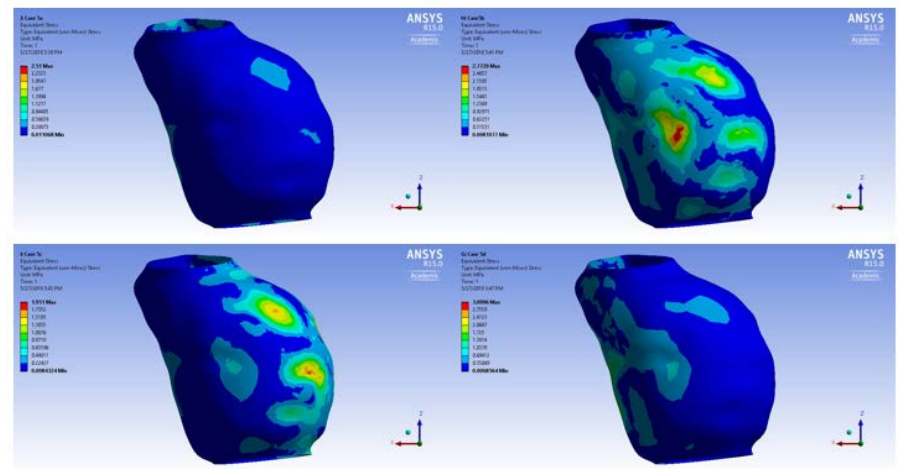

Figure 4 Effective wall stress distribution of four models at $Y$ plane

Four different models of AAA where von-Mises stress distribution across the walls are viewed across $Y$ plane can be seen in figure 4, where peak systolic pressure at static simulation. The maximum stress loca-tion is visible in second and third models with maximum stress value of $2.7729 \mathrm{MPa}$ and $1.951 \mathrm{MPa}$, respectively, equivalent to (von-Mises) stress, as AAA rupture is obvious to happen at the thinnest side of the wall.

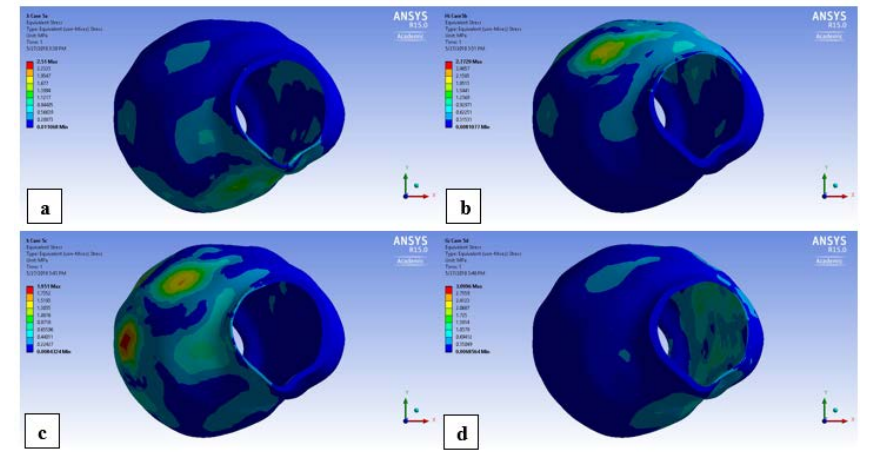

Figure 5 Effective wall stress distribution of four models at $X$ plane 
As seen in figure 5, four different models of AAA where von-Mises stress distribution across the walls are viewed across $Z$ plane, where peak systolic pressure at static simulation. The maximum stress location is only visible in third model (can be seen in figure $5 \mathrm{c}$ ) with a maximum stress value of $1.951 \mathrm{MPa}$ equivalent to (von-Mises) stress, as AAA rupture is obvious to happen at the thinnest side of the wall.

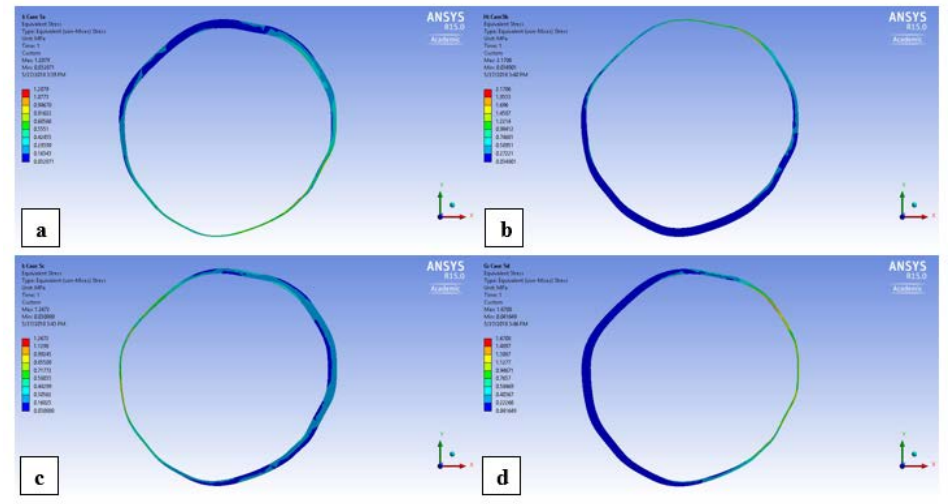

Figure 6 Effect of variable wall thickness on wall stress distribution of four models

The minimum and maximum stress at the thinnest side after slicing and meshing from the center of all the four models of AAA were found to be similar. In the first and second models, the thinnest side (at bottom for first model, see figure $6 \mathrm{~A}$ and top for second model, see figure $6 \mathrm{~B}$ ) exhibits a minimum stress level $0.40 \mathrm{MPa}$ and maximum stress level of 2.5 equivalent stress. Similarly, for the third and fourth models the thinnest side (at left for third model, see figure $5 \mathrm{C}$ and right for forth model, see fig-ure 5D) exhibits a minimum stress level $0.48 \mathrm{MPa}$ and maximum stress level of 2.5 equivalent stress.

\section{Discussion}

The aim of this study was to analyse the rupture among different models of AAA and compare their stress values at thin and thick sides of the models. Till date there has been no study with in-vitro rupture of AAA's with different position models constructed with the help of CAD software. In addition, many studies are earlier performed with computational analysis to understand the rupture of AAAs [9, 21-23]. Although all four models were designed using the ANSYS FEA technique there have been a different stress values recorded at the thinnest side. First two models with AAA thickness at anterior and posterior bears equal and higher amount of stress, while the thinnest side where rupture is most like-ly to occur have equal and less amount of stress. Moreover, when the thickness of AAA is at right and left display equal and higher amount of stress similar to earlier two models. However, the thinnest side exhibits an equal amount of stress but in contrast a higher stress values when compared with the first two models. Our findings and results strengthens the earlier studies performed by Polzer et al. (2010) [2] and Altuwaijri (2015) [3], where AAA wall stress was reduced by formation of thrombus. Thus reduc-ing the chance of AAA rupture and supporting the wall strength. However, a clinical study by Schurink et al. (2000) [24], contradicts our findings where formation of thrombus increases the chance of AAA rupture.

Our findings suggest that thrombus clearly works as a shield to guard the AAA wall from rupture. However, more research and further studies are required that corroborate the relation between the biological and mechanical factors to understand the role of the thrombus for AAA rupture. 
Omar Altwijri; Effect of Wall Variable Thickness on Patient Specific Finite Element Abdominal Aortic Aneurysm Models. Journal of Biomedical Engineering and Medical Imaging, Volume 5, No 5 October (2018), pp 6-12

\section{ACKNOWLEDGEMENT}

The authors extend their appreciation to the Deanship of Scientific Research at King Saud University for funding this work through the Research Project No NFG-14-02-16.

\section{REFERENCES}

[1] Di Martino, E.S., et al., Fluid-structure interaction within realistic three-dimensional models of the aneurysmatic aorta as a guidance to assess the risk of rupture of the aneurysm. Medical engineering \& physics, 2001. 23(9): p. 647-655.

[2] Polzer, S. and J. Bursa. Poroelastic model of intraluminal thrombus in FEA of aortic aneurysm. in 6th World Congress of Biomechanics (WCB 2010). August 1-6, 2010 Singapore. 2010. Springer.

[3] Altuwaijri, O. Finite Element Analysis OF Abdominal Aortic Aneurysms to Predict Risk of RuptureThe Role of The Thrombosis Thicknesses. in World Congress on Medical Physics and Biomedical Engineering, June 7-12, 2015, Toronto, Canada. 2015. Springer.

[4] Doyle, B.J., et al., An experimental and numerical comparison of the rupture locations of an abdominal aortic aneurysm. Journal of Endovascular Therapy, 2009. 16(3): p. 322-335.

[5] Vorp, D.A. and J.P.V. Geest, Biomechanical determinants of abdominal aortic aneurysm rupture. Arteriosclerosis, thrombosis, and vascular biology, 2005. 25(8): p. 1558-1566.

[6] McGloughlin, T.M. and B.J. Doyle, New approaches to abdominal aortic aneurysm rupture risk assessment: engineering insights with clinical gain. Arteriosclerosis, thrombosis, and vascular biology, 2010. 30(9): p. 1687-1694.

[7] Martufi, G., et al., Three-dimensional geometrical characterization of abdominal aortic aneurysms: image-based wall thickness distribution. Journal of biomechanical engineering, 2009. 131(6): p. 061015.

[8] Vorp, D.A., Biomechanics of abdominal aortic aneurysm. Journal of biomechanics, 2007. 40(9): p. 1887-1902.

[9] Fillinger, M.F., et al., Prediction of rupture risk in abdominal aortic aneurysm during observation: wall stress versus diameter. Journal of vascular surgery, 2003. 37(4): p. 724-732.

[10] Darling, R.C., et al., Autopsy study of unoperated abdominal aortic aneurysms. The case for early resection. Circulation, 1977. 56(3 Suppl): p. II161-4.

[11] Nicholls, S.C., et al., Rupture in small abdominal aortic aneurysms. Journal of vascular surgery, 1998. 28(5): p. 884-888.

[12] Thubrikar, M.J., J. Al-Soudi, and F. Robicsek, Wall stress studies of abdominal aortic aneurysm in a clinical model. Annals of vascular surgery, 2001. 15(3): p. 355-366. 
[13] Geest, J.P.V., et al., Towards a noninvasive method for determination of patient-specific wall strength distribution in abdominal aortic aneurysms. Annals of biomedical engineering, 2006. 34(7): p. 1098-1106.

[14] Raghavan, M.L., et al., Regional distribution of wall thickness and failure properties of human abdominal aortic aneurysm. Journal of biomechanics, 2006. 39(16): p. 3010-3016.

[15] J. Thubrikar, M.L., F. Robicsek, J. Al-Soudi, B. Fowler, M, Mechanical properties of abdominal aortic aneurysm wall. Journal of medical engineering \& technology, 2001. 25(4): p. 133-142.

[16] Raghavan, M.L., M.W. Webster, and D.A. Vorp, Ex vivo biomechanical behavior of abdominal aortic aneurysm: assessment using a new mathematical model. Annals of biomedical engineering, 1996. 24(5): p. 573-582.

[17] Van den Hengel, W.K. and F. van de Vosse, Abdominal aortic wall thickness and compliance. 2008.

[18] Truijers, M., et al., Wall stress analysis in small asymptomatic, symptomatic and ruptured abdominal aortic aneurysms. European Journal of Vascular and Endovascular Surgery, 2007. 33(4): p. 401-407.

[19] Fillinger, M.F., et al., In vivo analysis of mechanical wall stress and abdominal aortic aneurysm rupture risk. Journal of vascular surgery, 2002. 36(3): p. 589-597.

[20] Haller, C., et al., Sequential based analysis of Intima-Media Thickness (IMT) in common carotid artery studies. Atherosclerosis, 2007. 195(2): p. e203-e209.

[21] McGloughlin, T.M., et al., A finite element analysis rupture index (FEARI) as an additional tool for abdominal aortic aneurysm rupture prediction. 2009.

[22] Doyle, B.J., A. Callanan, and T.M. McGloughlin, A comparison of modelling techniques for computing wall stress in abdominal aortic aneurysms. Biomedical engineering online, 2007. 6(1): p. 38.

[23] Doyle, B.J., et al., Vessel asymmetry as an additional diagnostic tool in the assessment of abdominal aortic aneurysms. Journal of vascular surgery, 2009. 49(2): p. 443-454.

[24] Schurink, G., et al., Thrombus within an aortic aneurysm does not reduce pressure on the aneurysmal wall. Journal of Vascular Surgery, 2000. 31(3): p. 501-506. 